\title{
A PORTABle DEMONSTRATION OF ORBITS IN CURVED SPACE-TIME
}

\section{David B. Friend and Kevin Forkey}

Department of Astronomy and Department of Physics, Williams College, Williamstown, Massachusetts 01267, U.S.A.

Many introductory astronomy students, when confronted with the idea of curved space-time in discussions of relativity and cosmology, don't have a very good grasp of what this really means. They are told that the presence of mass "curves" space-time, but it is often not clear to them what is meant by that statement. We have developed a simple portable demonstration of what is meant by curved spacetime, by using a two-dimensional analog. We have stretched a thin rubber sheet over a circular metal frame which is supported a few inches above a table top. By placing a heavy weight (about a kilogram) in the center of the sheet, we can mimic the effect of the curvature of space-time around a massive object on an orbiting body (such as a planet orbiting a star). We roll a small ball around the sheet, and the ball describes approximately elliptical orbits around the weight (see the photograph). The orbits aren't exactly elliptical for two reasons: energy is dissipated by friction between the ball and the sheet, and the sheet doesn't quite have the correct shape (it is shaped roughly like $\ln r$ instead of $1 / r$ : see this by solving Laplace's equation in two dimensions with circular symmetry). We like to project this demonstration with a video camera placed directly above the sheet, so that the students can see the "orbit" without actually seeing the curvature of the sheet. This curvature is analogous to the actual 4 -dimensional structure of space-time: we can see the effect of the curvature without noticing the curvature itself. By letting the central mass

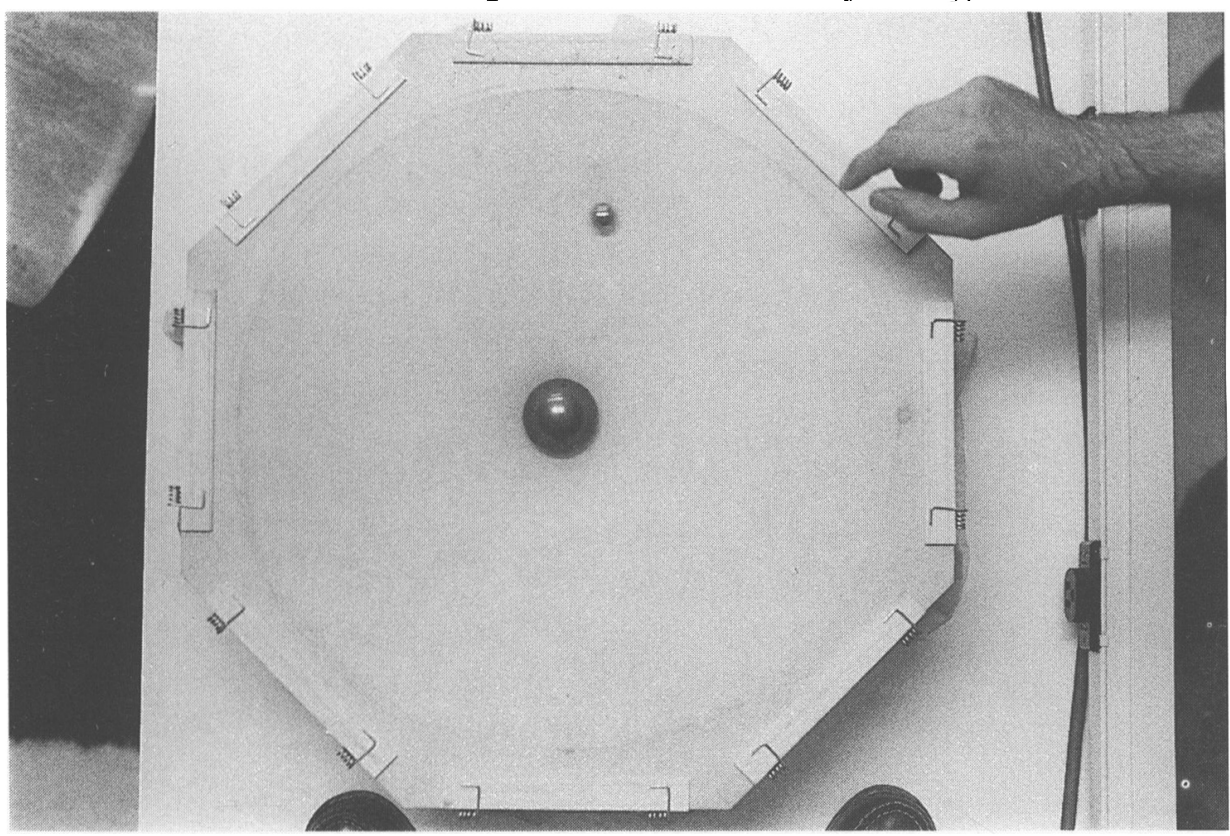

Figure 1. The demonstration of curved space-time 
be free to roll, we can also show how the more massive member of a binary system also moves in response to the gravitational field of (or the warping of space-time by) the smaller mass.

\section{DEMONSTRATIONS}

Participants were invited to present demonstrations that they found helpful in the teaching of astronomy. Two participants did so.

Roy L. Bishop (Acadia University, Canada) demonstrated the use of a small mirror for projecting an image of the sun on a screen. He recommends a piece of mirror, a few $\mathrm{mm}$ in diameter, mounted on a small stand. This combination can be placed on a window sill, or other sunny place, to catch the sunlight. Alternatively, a larger mirror can be used, if it is covered with cardboard or tape, with only a small area, a few $\mathrm{mm}$ in diameter, exposed. The screen should be located in a shadowed place. Note that the shape of the sun's image is not affected by the shape of the mirror!

Harry L. Shipman (University of Delaware, U.S.A.) described a new method for getting feedback from students. He attaches an answering machine to his telephone, and encourages students to "dial-a-prof" with comments or questions about the course work. Since the students can phone anonymously, they are less hesitant or embarrassed to do so. Questions left on the answering machine are dealt with at the next class. Harry finds this an effective way to determine which parts of the course are giving students most difficulty. 